\title{
Autoimmune Processes as an Important Parameter for the Pathogenesis of Aplastic Anemia and Paroxysmal Nocturnal Hemoglobinuria
}

Katja Kaastrup ${ }^{1}$, Kirsten Grønbæk², Sine Reker Hadrup ${ }^{3}$ and Andreas Glenthøj ${ }^{4 *}$

${ }^{1}$ Department for Immunology and Vaccinology, Technical University of Denmark, Denmark

${ }^{2}$ Rigshospitalet and Biotech Research and Innovation Centre BRIC, Denmark

${ }^{3}$ Department for Immunology and Vaccinology, Technical University of Denmark, Denmark

${ }^{4}$ Department of Hematology, Herlev Hospital, Denmark

\begin{abstract}
Aplastic anemia (AA) and paroxysmal nocturnal hemoglobinuria (PNH) are two rare and often concomitant hematologic diseases. Their development is - despite lack of decisive evidence - attributed autoimmune mechanisms. AA is characterized by cytopenias combined with a hypocellular bone marrow. The pathophysiology is believed to be immune-mediated with destruction of hematopoietic stem cells by autoreactive lymphocytes, a hypothesis supported by its response to immunosuppressive therapy.

A large proportion of patients with $\mathrm{AA}$ also have $\mathrm{PNH}$. In $\mathrm{PNH}$, somatic mutations in the PIGA gene in hematopoietic stem cells blocks synthesis of the glycosylphosphatidylinositol (GPI) anchor. As a result, blood cells deriving from the GPI-deficient clone lack GPI-anchored proteins, most notably the complement inhibiting factors CD55 and CD59. The clinical manifestations primarily arise because of uncontrolled complement activation on erythrocytes and thrombocytes.
\end{abstract}

This review summarizes current knowledge and theories about of the pathophysiology of both diseases with focus on immune mechanisms attributing to the two diseases.

Keywords: Aplastic anemia; Bone Marrow; Blood; Lymphocytes; T-cells; Autoimmune

\section{Introduction}

Aplastic anemia (AA) and paroxysmal nocturnal hemoglobinuria $(\mathrm{PNH})$ are hematopoietic disorders with distinctive disease mechanisms. However, they are often comorbid and both associated with an increased risk of developing clonal disease [1,2], suggesting a link between them.

AA is characterized by a markedly hypocellular bone marrow (BM) with few hematopoietic precursors. Consequently, hematopoiesis is inadequate, which leads to peripheral blood cytopenias [3,4]. Increasing amount of evidence suggest an immune mediated pathogenesis of AA leading to the BM failure (BMF) [5]. The immune dysregulation is marked by an increased activation of auto reactive lymphocytes together with an impaired immune suppression.

The management of AA has drastically improved since the 1970s with the introduction of allogeneic stem cell transplantation and immunosuppressive therapy (IST), as well as optimization of supportive care [6]. Development of late clonal diseases, such as PNH and myelodysplastic syndrome (MDS) has occasionally been reported for patients successfully treated with IST [1].

$\mathrm{PNH}$ is an acquired clonal disorder in hematopoietic stem cells (HSCs), resulting in a defect in the synthesis of the glycosylphosphatidylinositol (GPI) anchor. Genetically, almost all described cases are attributed to somatic mutations in the phosphatidylinosityl glycan class A gene (PIGA gene) [7], which encodes a protein essential for synthesis of GPI anchors (GPI-A). Clinically, PNH is characterized by haemolytic anemia, thrombosis, and variable degrees of BMF [7-11]. Hemolysis and thrombosis is thought to be due to a deficiency in the GPI anchored proteins (GPI-AP) CD55 and CD59, both being complement inhibitors. This deficiency, together with reduced fibrinolysis, NO scavenging and the presence of free hemoglobin, results in a chronic complement-mediated hemolysis as well as activation of thrombocytes [7]. The humanized monoclonal antibody, Eculizumab, inhibits the complement factor 5 and thereby the formation of the membrane attack complex (MAC). It is, in developed countries, the first choice of treatment for $\mathrm{PNH}$, although $\mathrm{BM}$ transplantation (BMT) remains the only cure [7].

The pathogenesis of both $\mathrm{AA}$ and $\mathrm{PNH}$ are complex and the disease mechanism making them comorbid is yet to be elucidated. This review provides an overview of the immune mechanisms involved in the development of both diseases and summarizes the current understandings of the disease mechanisms linking them together.

\section{Immune Mechanisms Involved in Development of Aplastic Anemia}

\section{Impaired immune suppression}

$\mathrm{CD}^{+} \mathrm{CD} 25^{+} \mathrm{FOXP}^{+}{ }^{+}$Regulatory $\mathrm{T}$ cells (Tregs) are believed to control development and progression of autoimmunity by suppressing autoreactive effector $\mathrm{T}$ cells. AA patients show dysregulation of this immunosuppressive mechanism: First, they have significantly decreased numbers of Tregs compared to healthy controls both in peripheral blood $(\mathrm{PB})$ and $\mathrm{BM}$ [12], possibly resulting from of altered transcription of FOXP3 [13]. Secondly, Tregs of AA patients

*Corresponding author: Andreas Birkedal Glenthøj, Department of Hematology, Herlev Hospital, Herlev Ringvej 752730 Herlev, Tel: +45 40266 997; Fax: +45 44532518; E-mail: andreas@hematology.dk

Received March 22, 2018; Accepted June 11, 2018; Published June 18, 2018

Citation: Kaastrup K, Grønbæk K, Hadrup SR, Glenthøj A (2018) Autoimmune Processes as an Important Parameter for the Pathogenesis of Aplastic Anemia and Paroxysmal Nocturnal Hemoglobinuria. J Blood Lymph 8: 215. doi:10.4172/21657831.1000215

Copyright: (c) 2018 Kaastrup K, et al. This is an open-access article distributed under the terms of the Creative Commons Attribution License, which permits unrestricted use, distribution, and reproduction in any medium, provided the original author and source are credited. 
show decreased ability to suppress autoreactive T cells [12]. Figure 1 summarizes the events leading to development of AA. An impaired T cell regulation and suppression by regulatory $\mathrm{T}$ cells (Tregs) leads to an increased $\mathrm{T}$ cell activation, seen as an increase in cytokines specific for the type 1 immune response. This activation and production of the specific cytokines results in killing of hematopoietic stem cells $\left(\mathrm{CD} 34^{+}\right.$ cells) and consequently impaired hematopoiesis and cytopenias.

IL-35 has been identified as a potentially immunosuppressive cytokine produced by Treg cells and is required for maximum suppressive activity [14]. It has been shown to inhibit the proliferation capacity of PBMCs and induce a significant reduction in the absolute number of $\mathrm{CD}^{+}$and $\mathrm{CD} 8^{+}$effector $\mathrm{T}$ cells in both healthy controls and AA patients [15]. The level of IL-35 is significantly lower in AA patients than in healthy controls [15], suggesting a mechanism for the impaired suppressive capacity of Tregs in AA.

Tregs modulate the activity of a wide variety of effector $\mathrm{T}$ cells by cell contact inhibition, which depends on their ability to move into physical proximity with their targets by homing to specific tissues [16]. The stromal cells in the BM strongly express CXCL12, the ligand for CXCR4. Tregs home to and are retained in the BM by signalling through CXCR4/SDF1a, a CXCL12 chemokine [17]. Studies show that Tregs from patients with AA have less migratory capacity toward SDF1a, compared with Tregs from healthy controls [12].

The impaired migratory potential of Tregs is likely due to a lower expression of CXCR4 mRNA in PB and BM Tregs from patients, compared to controls [12], since there are comparable levels of SDF$1 \alpha$ in BM fluid from controls and patients. This suggests that the attenuation of chemotactic signals is not responsible for the impaired migratory capacity [12].

\section{T cell mediated suppression of hematopoiesis}

A hypocellular BM is the hallmark of AA and may be attributed to $\mathrm{T}$ cell mediated BM suppression [5]. There is substantial evidence supporting this, such as hematologic reconstitution after IST with drugs targeting T cell function [18] and improvement of in vitro colony formation after removal of $\mathrm{T}$ lymphocytes from the marrow of AA patients [19].

$\mathrm{BM}$ failure might be due to a stem cell lesion or to an inefficient stromal microenvironment. The latter does not seem to be the case in most of AA as factors stimulating hematopoiesis are usually normal or elevated in patients [20].

Early experiments demonstrate that BM lymphocytes from AA patients suppress normal haematopoiesis. Stem cells and primitive progenitor cells can be identified by the expression of the cytoadhesive protein $\mathrm{CD} 34$ and quantified by flow cytometry. The number of $\mathrm{CD} 34^{+}$cells is greatly reduced in AA. It has been shown that normal differentiation can be obtained following lymphocyte depletion and that such patient-derived lymphocytes directly inhibit haematopoiesis in co-culture experiments $[16,19]$.

Activated lymphocytes that produce IFN- $\gamma$ and TNF- $\alpha$ are found in peripheral blood of AA patients and there are significantly higher percentages of cytolytic PB- and BM T cell clones in AA patients than in controls [21]. Furthermore, production of INF- $\gamma$, TNF- $\alpha$ and IL-2 is increased in T-cells from patients with AA [15,22-24] suggest an ongoing Th1 response that may be responsible for the human stemand progenitor cell (HSPC) destruction, as depicted in Figure 2. (1) T cells recognize an epitope, possibly a self-peptide, on the hematopoietic stem and progenitor cells (HSPC), this leads to an (2) expansion of
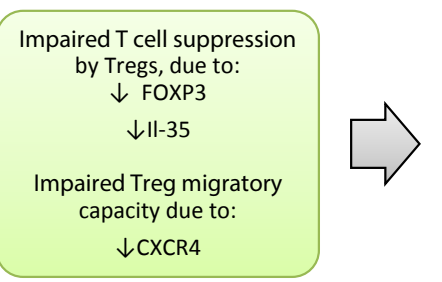

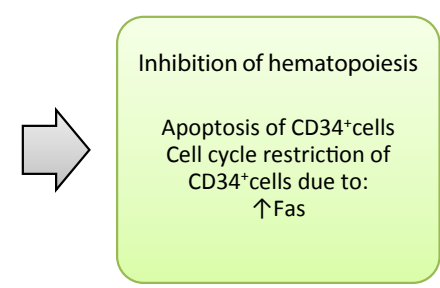

Figure 1: Summary of events that may lead to aplastic anemia (AA)

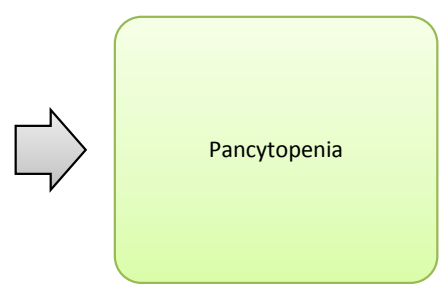

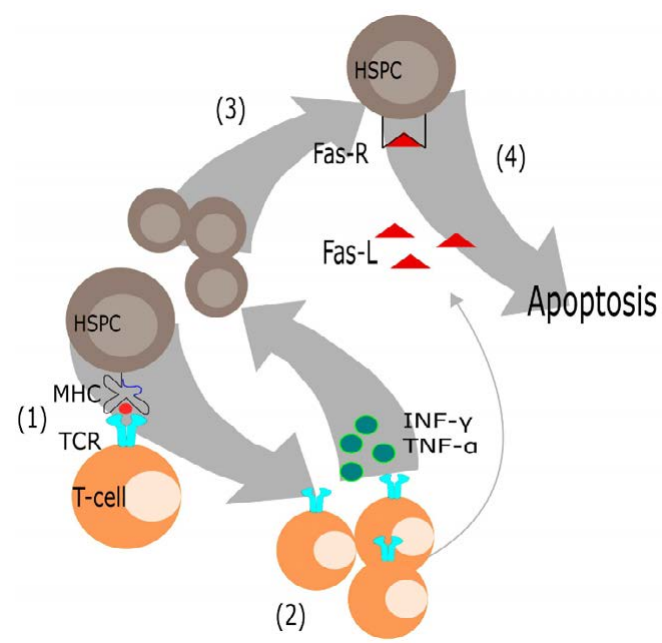

Figure 2: Possible mechanism of an autoimmune mediated bone marrow failure in aplastic anemia. 
autoreactive T cells. Production of INF- $\gamma$ and TNF- $\alpha$ suggest that the HSPCs are suppressed through a Th1 T cell response. (3) The cytokines induce expression of Fas receptor (Fas-R) on the HSPCs. The autoreactive $\mathrm{T}$ cells induce apoptosis of HSPCs by releasing a Fas ligand, that binds and activate the Fas-R, thus (4) mediating intracellular signal transduction leading to apoptosis.

The transcription factor T-bet binds to the INF- $\gamma$ promotor and is responsible for the Th1 phenotype. T-bet is also up-regulated in AA patients [25] which correlates with the increased expression of INF- $\gamma$ [25].

Th1 cells assist in activation of $\mathrm{CD}^{+} \mathrm{T}$ cells by direct interaction or licensing of dendritic cells and expression of IL-2 that drives the $\mathrm{CD}^{+} \mathrm{T}$ cell proliferation. $\mathrm{PB}$ mononuclear cells from patients with AA produce large amounts of IL-2 in vitro, and their circulating T cells have high expression the IL-2 receptor $[24,26]$. This suggests an activation of $\mathrm{CD}^{+} \mathrm{T}$ cells.

The suppression of normal hematopoiesis by addition of patient's $\mathrm{BM}$ to normal marrow, the low $\mathrm{CD} 34^{+}$cell number, and the elevated levels of type 1 cytokines all supports a disease mechanism involving a T cell mediated BM suppression. INF- $\gamma$ and TNF- $\alpha$ suppress the proliferation of early and late HSPCs and exerts a direct inhibitory effect on hematopoiesis $[23,27,28]$. The BM suppression by these cytokines, may occur not only as an inhibition of hematopoietic cell proliferation, but also by apoptotic depletion of HSPCs [28].

Both IFN- $\gamma$ and TNF- $\alpha$ induce expression of the Fas-receptor on $\mathrm{CD} 4^{+}$cells in vitro [29]. Patients with active disease shows higher level of Fas expression than those who recover [29]. A monoclonal anti-Fas antibody, mediates apoptosis upon binding to Fas antigen [30]. However, expression of Fas ligand (Fas-L) on the surface of $\mathrm{CD}^{+}$ T-cells is not detectable in AA patients, but higher levels of intracellular FasL are found in the T cells from AA patients compared to T cells from normal controls [31]. The proportion of apoptotic cells is significantly increased in BM biopsies from AA patients [32].

The reduction of $\mathrm{CD} 34^{+}$cells and their failure to form colonies may be related to a block of cell cycling and arrest in the $G_{0}$ phase [3]. In normal BM $25-40 \%$ of CD34 cells are in $\mathrm{G}_{1}$ or S-phase [3]. It has not yet been possible to analyse cell cycle directly on $\mathrm{CD} 34^{+}$cells from AA due to the patients' hypocellular BM. However, it has been shown that cycle status is associated with the expression of c-kit [3], a receptor tyrosine kinase protein. The percentage of c-kit ${ }^{+}$cells within the CD34 ${ }^{+}$ cell population is significantly decreased compared to controls, which is consistent with the decreased number of mitotically active CD $34^{+}$ cells [3].

\section{Antigen recognition in bone marrow}

Several studies document the presence of an oligoclonal $\mathrm{T}$ cell response in vivo [33] and a cytotoxic activity of these $\mathrm{T}$ cell clones on autologous HSPCs in vitro. For lymphocytes to be activated they must recognize and bind the proper antigen. However, the initial antigen exposure that mediate the immune response in idiopathic AA is yet unknown.

The fact that patient BM cells suppress normal differentiation and hematopoiesis by their addition to normal BM cells [9] imply that the antigen recognition is shared between humans. It may be that $\mathrm{T}$ cells recognize the self-peptides with low affinity, but get activated due to the impaired immune suppression. However, the dysregulatory events leading to loss of tolerance are unknown.
Studies have shown the presence of leukocytes with a copy-number neutral loss of heterozygosity in the short arm of chromosome 6 (6pUPD) [34,35]. 6pUPD is commonly involved in the HLA locus, leading to the loss of one HLA haplotype [34]. This could represent an escape of HSPCs with 6pUPD from the attack of CTLs that are specific to autoantigens presented by the lacked HLA type. HLA-B ${ }^{\star} 40: 02$ have been revealed to be the most frequently lost allele [36]. The high prevalence of HLA-B ${ }^{\star} 40: 02$ loss suggest that antigen presentation by HSPCs to CTLs via the HLA-B allele plays an important role in the pathogenesis of AA.

\section{Immune Manipulation as Treatment of AA}

Hematopoiesis can be restored in most cases in SAA with HSCT or IST. HSCT is used as first-line treatment for patients under 40 years of age and who has an HLA-compatible sibling donor [37].

A large study from 1991-2004 investigated the impact of age on outcomes after HSCT using HLA-matched sibling donors and found that the 5 -year probability of overall survival is $72 \%$ in patients between $20-40$ years and only $53 \%$ in patients over 40 years old [38].

IST is used as treatment for AA when HSCT are not feasible. The standard IST combination is antithymocyte globulin (ATG) and cyclosporine A (CsA), which produces hematologic recovery in $60-70 \%$ of cases [18]. Recently, a phase 1-2 study has shown that addition of the thrombopoietin agonist, eltrombopag, to IST likely improves outcomes [39]. A common complication of AA after IST is the progression to myelodysplastic syndrome or acute myeloid leukemia.

ATG binds to lymphocytes [40] and temporarily deplete T-cells in peripheral blood and to lesser extent in lymph nodes [41]. The mechanism of action is both activation-associated lymphocyte apoptosis by ATG-triggered Fas-ligand and Fas expression ${ }^{41}$ and complement-dependent lysis $[42,43]$. CsA inhibits $\mathrm{T}$ cell activation and proliferation by blocking the transcription of cytokine genes [44]. CsA binds to and inhibit calcineurin, an enzyme that activates the transcription factor NF-AT [44] important for expression of several effector cytokines. The immunosuppressive effect of CsA is attributed to the blockage of the c-jun-N-terminal kinase- and p38 pathway [44].

\section{Immune mechanisms involved in development of paroxysmal nocturnal hemoglobinuria}

PNH is characterized by three clinical hallmarks: Thrombosis, intravascular hemolysis, and variable degrees of BMF. The disease starts with a clonal expansion of HSPCs that have genetically lost their capacity to produce glycosylphosphatidylinositol (GPI), a glycolipid that anchors many proteins to the cell surface [10]. Figure 3 summarized the events leading to the development of PNH. A mutation in the PIGA gene in hematopoietic stem and progenitor cells (HSPCs) result in an impaired GPI anchor (GPI-A) synthesis and thus GPI-A deficient HSPC clones. An autoimmune attack that selectively target GPI+ HSPCs favors the clonal expansion of GPI deficient HSPCs. Such an escape may be mediated by an impaired NK cell activation through the absence of stress induced UL-binding proteins (ULBPs), which is a GPI-A protein, or because the autoimmune attack is attacking the GPI molecule itself - thereby favoring the expansion of GPI- HSPCs. The GPI- erythrocytes originating from the GPI- HSPCs are susceptible to hemolysis and thrombosis due to increased complement activation.

\section{Cellular effects}

The expansion of GPI- HSPCs leads to a GPI anchor protein (GPI- 


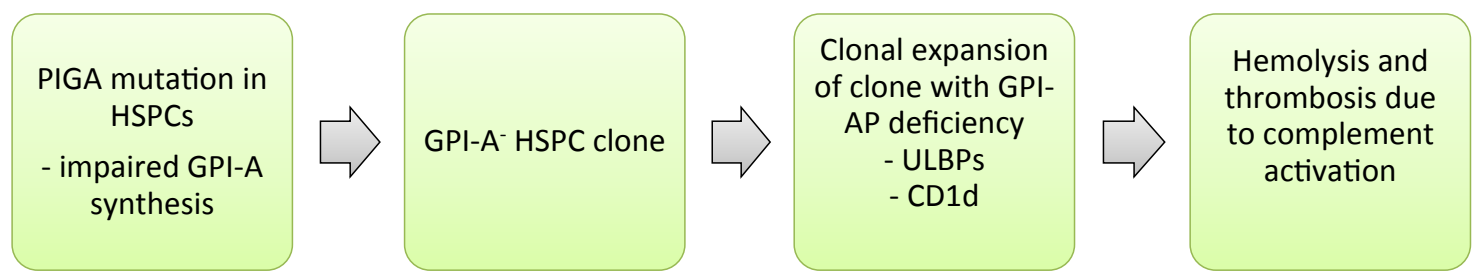

Figure 3: Summary of events that may lead to paroxysmal nocturne hematuria $(\mathrm{PNH})$.

AP) deficiency in all blood cells. This leads to chronic complement activation from the alternative complement pathway, through a deficiency of the complement-inhibitor proteins CD55 and CD59. The GPI-AP CD55 inhibits complement activation by preventing the intrinsic assembly of C3 and C5 convertases [45]. The GPI-AP CD59 inhibits the membrane attack complex (MAC) formation by reducing the polymerization of $\mathrm{C} 9$. It binds to $\mathrm{C} 8$ in the C5b-8 complex and prevent binding of C9 [46]. Furthermore, it binds to C9 in the C5b-9 complex, preventing recruitment of additional C9 molecules [46].

\section{Genetics}

GPI biosynthesis is a post-translational event that occurs in the endoplasmic reticulum [47]. Most patients with $\mathrm{PNH}$ has a defect in the first step of GPI synthesis [48]. Theoretically, any mutation of any gene in the pathway could lead to PNH. However, the first gene in the pathway, the PIGA gene, is an X-linked gene. Thus, a single somatic mutation of the PIGA gene is sufficient to produce a PNH phenotype. This also applies in women as one of their two alleles has already been silenced by X chromosome inactivation [49]. The other known genes involved in the GPI-A pathway are all autosomal. In consequence, a mutation on both alleles would be required to interrupt the biosynthesis [7].

The pathogenesis of PNH was elegantly revealed in an elegant in vitro study in which the PIGA gene was transfected into GPI-A deficiency lymphocytes from a patient with $\mathrm{PNH}$, hereby restoring the surface expression of GPI-AP [50].

There are no mutational hotspots, although many mutations occur on exon 2, the largest exon of the PIGA gene [51]. Most PIGA mutations are small insertions or deletions, which result in a frameshift in the coding region, leading to a truncated protein [50,51]. The PIGA mutations must occur in a HSCs and must achieve clonal dominance, for it to cause PNH [52].

A few cases of patients with homozygous mutations on the PIGT gene [53], coding for the protein responsible for catalysing the translocation of the protein unto the GPI-A, have been reported [54].

\section{Clonal expansion of PNH stem cells}

The PIGA mutation alone is not sufficient to cause $\mathrm{PNH}$, since small clones with PIGA mutations are commonly found in healthy individuals without causing disease.

The mechanisms leading to clonal expansion and dominance of $\mathrm{PNH}$ stem cells remain a topic of debate. In principle, two mechanisms can lead to the expansion. The expansion may occur from an acquired mutation other than the PIGA mutation, leading to a growth advantage for the mutated clone.

The leading hypothesis, however, based on the close clinical relationship between $\mathrm{PNH}$ and $\mathrm{AA}$, is that an autoimmune attack, that selectively target $\mathrm{GPI}^{+}$HSPCs, favours expansion of GPI- HSPCs $[7,56,57]$. This would happen, if the auto-antigen was a GPI-AP.

There is increasing amount of evidence supporting this. Phenotypically, GPI-AP- $\mathrm{CD}^{+} 4^{+}$cells show similar proliferation capacity in vitro with normal control $\mathrm{CD}_{3} 4^{+}$cells from healthy individuals [58] which suggest that $\mathrm{PNH}$ clones do not have an intrinsic growth advantage. Furthermore, the fact that $\mathrm{PNH}$ clones are found in low levels in healthy individuals [55] and that these clones do not have an intrinsic property to overgrow normal cells [59], suggest that an extrinsic selective pressure determines the expansion of the $\mathrm{PNH}$ clone.

Evidence supporting an autoimmune attack on the BM is that most patients with PNH show high level of oligoclonality in terms of their T-cell receptor beta chain usage [9]. The antigen binding sites of the TCR are composed of the hyper variable complementarity determine regions (CDR). The sequence and length of CDR3 represents a specific $\mathrm{T}$ cell clonotype and CDR3 size analysis in $\mathrm{PNH}$ patients shows a nongaussian distribution of CDR3 length and thus an oligoclonal $\mathrm{T}$ cell population [9].

Giving the large diversity of the physiological TCR repertoires, the detection of such identical or quasi-identical clonotypes in multiple $\mathrm{PNH}$ patients suggests an immune response driven by the same antigen. Such a selective pressure could be mediated by an immune attack on the BM. NK, NKT, and T cells are all feasible candidates for effector lymphocytes that damage BM cells. How these cells might selectively target $\mathrm{GPI}^{+} \mathrm{HSPCs}$ is still not clear.

Evidence suggests that stress-induced UL-binding proteins (ULBPs) are involved in the activation of NK cells in PNH, by acting as a ligand for its receptor, NKG2D as shown in Figure 4. GPI- HSPCs do not express ULBPs on their cell surface, since it is a GPI anchored protein. The absence of ULBPs may lead to an impaired NK cell activation, thus favoring the expansion of GPI- HSPCs.

ULBPs are GPI-A proteins and are differentially expressed on $\mathrm{GPI}^{+}$ cells, but not on GPI cells [60]. The absence of ULBPs results in an impaired NK activation. This is shown by the fact that GPI- K562 cells are less susceptible to NK cells than $\mathrm{GPI}^{+} \mathrm{K}_{562}$ cells [60]. Likewise, antibodies to NKG2D inhibit the killing and abolished the difference in the killing of $\mathrm{GPI}^{+} \mathrm{K} 562$ cells and GPI ${ }^{-}$K562 cells [60]. Transferred to in vivo settings, PNH cells-also lacking ULBPs would also be relatively spared from NK-mediated killing.

Another theory is that the target of the autoimmune attack may be the GPI molecule itself rather than a GPI-linked protein. GPI is thought to be a natural ligand to CD1d [61], a molecule presenting lipid antigens and is important in NKT cell activation. A study show that CD1d-restricted T cells are expanded in some patients [56]. CD1d is expressed on HSPCs [62] and GPI may be a CD1d-ligand. This suggests that GPI itself is the target of the autoimmune attack, as depicted in Figure 5. The GPI molecule itself may presented to NKT cells by CD1d, 


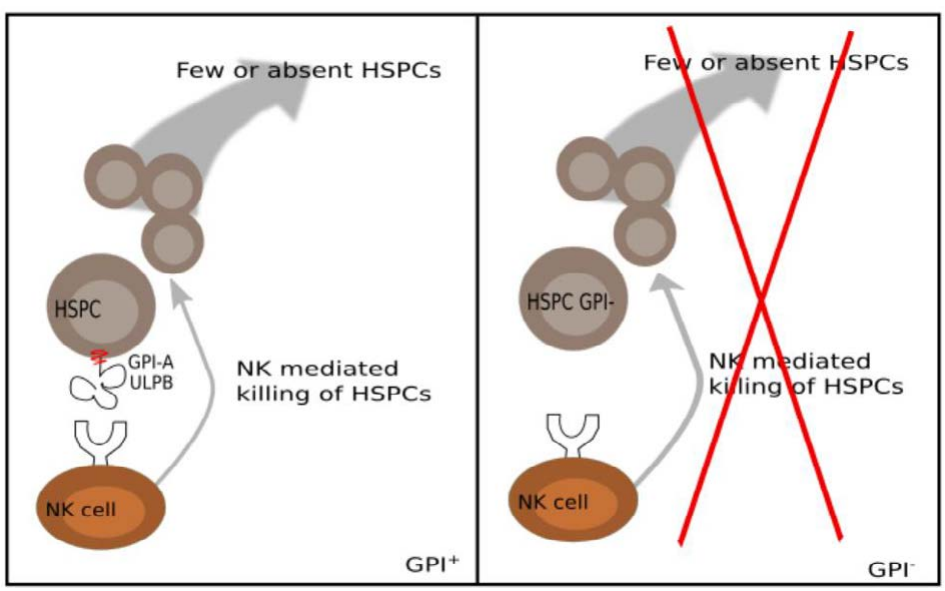

Figure 4: Possible mechanism of impaired natural killer cell activation by GPI deficient hematopoietic stem and progenitor cells (GPI- HSPCs).

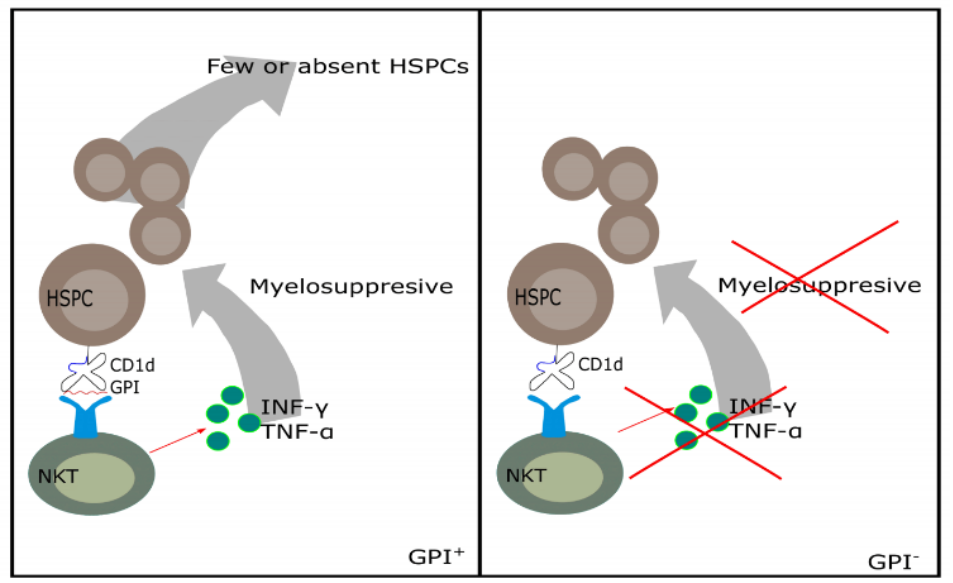

Figure 5: Possible mechanisms showing how GPI-deficient hematopoietic stem and progenitor cells (GPI-HSPCs) expand in paroxysmal nocturne hemoglobinuria by avoiding NKT cell activation.

leading to an activation and killing of GPI+ HSPCs leading to the expansion of GPI- HSPCs. This would explain why the GPI-deficient HSPCs expand in PNH.

\section{Immune Manipulation as Treatment of PNH}

Eculizumab, a terminal complement inhibitor, and BMT remains the only widely effective therapies for patients with classic PNH. Giving the risk of transplant-related morbidity [63], Eculizumab is the first line of treatment. However, if Eculizumab is not available or effective, BMT remains a viable - and potentially curative - treatment of classic PNH. Since Eculizumab is only active against the haemolytic component of $\mathrm{PNH}$, concomitant bone marrow failure such as myelodysplasia or AA would in themselves provide indication for BMT in fit patients.

Eculizumab is a humanized monoclonal antibody that binds to complement $\mathrm{C} 5$, preventing its activation to $\mathrm{C} 5 \mathrm{~b}$ and hereby inhibits the formation of MAC. Essentially, this compensates for CD59 deficiency in $\mathrm{PNH}$ and prevents intravascular hemolysis and its clinical consequences such as smooth muscle dysfunction and increased thrombocyte aggregation $[7,64]$. However, it does not block for the activity of $\mathrm{C} 3$ convertase and does therefore not compensate for the CD55 deficiency of PNH cells. This means that the PNH cells are still susceptible to extravascular hemolysis, which often is detected with anti-C3d positivity on a direct antiglobulin test (DAT) (36).

Eculizumab has no effect on the stem-cell abnormalities and only treats the hemolysis associated symptoms, rather than curing the disease, meaning that indefinite treatment is needed. Eculizumab is extremely expensive and have several side effects. Patients treated with Eculizumab are in increased risk of meningococcal infections and should undergo meningococcal vaccination prior to the first treatment with Eculizumab [65,66].

\section{Progression to malignant diseases}

$\mathrm{AA}$ and $\mathrm{PNH}$ are closely associated diseases and and many patients with AA have a PNH clone or may develop such a clone over time. a condition known as AA/PNH syndrome. Such a comorbidity is seen in approximately $40-50 \%$ of cases [67]. Patients with either AA or PNH are predisposed to MDS and the risk may increase after IST $[1,68,69]$.

The frequent development of AML and MDS is associated with clonal haematopoiesis $(\mathrm{CH})$ seen in many patients with AA [70]. Nonetheless, the mechanisms underlying malignant transformation in AA patients without proof of $\mathrm{CH}$ is yet to be determined. There seems to be at least two types of genetic alterations involved in the 
Citation: Kaastrup K, Grønbæk K, Hadrup SR, Glenthøj A (2018) Autoimmune Processes as an Important Parameter for the Pathogenesis of Aplastic Anemia and Paroxysmal Nocturnal Hemoglobinuria. J Blood Lymph 8: 215. doi:10.4172/2165-7831.1000215

clonal selection in AA; (1) mutations in epigenetic regulators that are commonly seen in MDS/AML, such as DNMT3A and ASXL1 and genetic alterations in genes highly specific or overrepresented in AA such as mutations in PIGA/BCOR/ BCORL1 genes and uniparental disomy of the $6 \mathrm{p}$ arm (6pUPD). Distinction between AA and hypoplastic MDS is notoriously difficult and it is possible that AA with MDS/AML associated mutations would be better classified as hypoplastic MDS, whereas patients with PIGA/BCOR/BCORL1 mutation would represent patients with genuine autoimmune driven AA. This is underscored by the observation that MDS/AML mutations is associated with progression of AA to MDS/AML [70].

In a large study of samples from 439 patients with AA targeted deep sequencing identified a total for 249 somatic mutations among 159 patients $(36 \%)$. The most frequently mutated genes were BCOR and BCORL1 (9.3\% of the patients), PIGA (7.5\%), DNMT3A $(8.4 \%)$ and ASXL1 (6.2\%) [71]. The presence and number of mutations on DNMT3A and ASXL1 correlated positively with age [71]. 6pUPD was found in $13 \%$ of patients and in total $\mathrm{CH}$ was identified in $47 \%$ of AA patients [71].

These data suggest that $\mathrm{CH}$ in AA is highly likely to represent the early stages of leukemogenesis. Furthermore, the data show a parallel between BMF and normal aging by the age-related spontaneous conversion of methylated cytosine to thymidine. The exact mechanism of selection of mutated cells in AA is still unknown, however it can be speculated whether the treatment with immunosuppressive agents may in some contexts allow for expansion of the mutated clone, probably after accumulation of additional genetic events [72].

\section{Main Findings of the Literature}

\section{Aplastic anemia (AA)}

- AA is characterized by a hypocellular bone marrow (BM). Research suggest an immune mediated pathogenesis of AA leading to the BM failure [5].

- There is a decreased number of Regulatory T cells (Tregs) in both peripheral blood and BM of patients compared to healthy individuals [12].

- The Tregs from AA patients have a decreased ability to suppress autoreactive T cells [12]

- There is an increased activation of lymphocytes and production of INF- $\gamma$, TNF- $\alpha$ and IL-2 in AA patients, suggesting an ongoing Th1 response [15,21-24].

- Loss of heterogenicity of the short arm on chromosome6 (6pUPD) is commonly involved, with HLA- $\mathrm{B}^{\star} 40: 02$ being the most frequently lost allele [34-36].

\section{Paroxysmal nocturnal hemoglobinuria (PNH)}

- Most PNH patients has a defect in the first step of the GPI synthesis as a result of a mutation in the PIGA gene, leading to hemopoietic stem progenitor cells (HSPC) with a GPI deficiency (GPI-) [48].

- The leading hypothesis of the mechanisms leading to clonal expansion of the $\mathrm{PNH}$ stem cell is that an autoimmune attack, that selectively target GPI+ HSPCs, favors expansion of GPIHSPCs $[7,56,57]$.

- Most patients with PNH show high level of oligoclonality in terms of their TCR beta chain usage [9].
- The expansion of GPI- HSPC leads to a GPI anchor protein (GPI-AP) deficiency in all blood cells and thus impaired inhibition of the complement system through the GPI-APs CD55 and CD59, leading to hemolysis [45,46].

\section{Discussion}

The diagnosis of AA still relies on careful examination of a bone marrow examination and peripheral blood cell counts. Often, the distinction of AA from hypoplastic MDS is dubious and relies on subtle changes in morphology in the bone marrow. The identification of somatic mutations in a large proportion of AA patients give rise to the notion that these patients could be regarded as having MDS as demonstrated by their increased propensity to develop obvious MDS and progress to AML [70]. On the other hand, a significant proportion of MDS patients display features of autoimmunity such as 6pUPD [73] and response to IST [74]. AA is strongly associated with the presence of a PNH clone. This is hardly a coincidence and suggests common underlying immune dysregulation that specifically affect the HSPCs and that the acquirement of PIG-A mutations could allow the HSPCs to escape this immune dysregulation, thereby evolving into $\mathrm{PNH}$. Progress in the understanding of the underlying molecular mechanisms of AA as well as PNH should allow future diagnostic classification to be shifted from morphology to immunological and genetic features.

\section{Conflicts of Interest and Source of Funding}

$A G$ has received an unrestricted grant from Alexion Pharmaceuticals. For the remaining authors, no relevant conflicts of interest were declared.

\section{References}

1. Li Y, Li X, Ge M, Shi J, Qian L, et al. (2011) Long-term follow-up of clonal evolutions in 802 aplastic anemia patients: a single-center experience. Ann Hematol 90: 529-537.

2. Ogawa S (2016) Clonal hematopoiesis in acquired aplastic anemia. Blood 128 337-347.

3. Maciejewski JP, Anderson S, Katevas P, Young NS (1994) Phenotypic and functional analysis of bone marrow progenitor cell compartment in bone marrow failure. $\mathrm{Br} \mathrm{J}$ Haematol 87: 227-234.

4. Young NS (2002) Acquired Aplastic Anemia. Ann Intern Med 136: 534-546.

5. Epstein FH, Young NS, Maciejewski J (1997) The Pathophysiology of Acquired Aplastic Anemia. N Engl J Med 336:1365-1372.

6. Young NS, Calado RT, Scheinberg P (2006) Current concepts in the pathophysiology and treatment of aplastic anemia. Blood 108: 2509-2519.

7. Brodsky RA (2014) Paroxysmal nocturnal hemoglobinuria. Blood 124: 2804 2811.

8. MacPherson S, Kondejewski JM (2015) Paroxysmal Nocturnal Hemoglobinuria Pocket Guide ( $1^{\text {th }}$ edn)

9. Gargiulo L, Lastraioli S, Cerruti G, Serra M, Loiacono F, et al. (2007) Highly homologous T-cell receptor beta sequences support a common target for autoreactive T cells in most patients with paroxysmal nocturnal hemoglobinuria. Blood 109: 5036-5042.

10. Rachidi S, Musallam KM, Taher AT (2010) A closer look at paroxysma nocturnal hemoglobinuria. Eur J Intern Med 21: 260-267.

11. de Muckadell OBS, Haunsø S, Hansen NE (2004) Medicinsk Kompendium Bind $2\left(16^{\text {th }}\right.$ edn), Nyt Nordisk Forlag.

12. Shi J, Ge M, Lu S, Li X, Shao Y, et al. (2012) Intrinsic impairment of CD4(+) CD25(+) regulatory $T$ cells in acquired aplastic anemia. Blood 120: 1624-1632.

13. Solomou EE, Rezvani K, Mielke S, Malide D, Keyvanfar K, et al. (2007) Deficient CD4+ CD25+ FOXP3+ T regulatory cells in acquired aplastic anemia. Blood 110: 1603-1606

14. Collison LW, Workman CJ, Kuo TT, Boyd K, Wang Y, et al. (2007) The inhibitory cytokine IL-35 contributes to regulatory T-cell function. Nature 450:566-569. 
Citation: Kaastrup K, Grønbæk K, Hadrup SR, Glenthøj A (2018) Autoimmune Processes as an Important Parameter for the Pathogenesis of Aplastic Anemia and Paroxysmal Nocturnal Hemoglobinuria. J Blood Lymph 8: 215. doi:10.4172/2165-7831.1000215

15. Yu W, Ge M, Lu S, Shi J, Li X, et al. (2015) Anti-inflammatory effects of interleukin-35 in acquired aplastic anemia. Cytokine 76: 409-416.

16. Campbell DJ, Koch MA (2011) Phenotypical and functional specialization of FOXP3+ regulatory T cells. Nat Rev Immunol 11: 119-130.

17. Zou L, Barnett B, Safah H, Larussa VF, Evdemon-Hogan M, et al. (2004) Bone Marrow Is a Reservoir for CD4+CD25+ Regulatory T Cells that Traffic through CXCL12/CXCR4 Signals. Cancer Res 64: 8451-8455.

18. Scheinberg P, Young NS (2012) How I treat acquired aplastic anemia. Blood 120: $1185-1196$

19. Kagan WA, Ascensao JA, Pahwa RN, Hansen JA, Goldstein G, et al. (1976) Aplastic anemia: presence in human bone marrow of cells that suppress myelopoiesis. Proc Natl Acad Sci U S A 73: 2890-2894

20. Kojima S, Matsuyama T, Kodera Y (2017) Hematopoietic Growth Factors Released by Marrow Stromal Cells From Patients With Aplastic Anemia. Blood J 79: 2256-2261.

21. Viale M, Merli A, Bacigalupo A (1991) Analysis at the clonal level of T-cell phenotype and functions in severe aplastic anemia patients. Blood 78: 12681274.

22. Martínez-Jaramillo G, Flores-Figueroa E, Gómez-Morales E, Sánchez-Valle E, Mayani $H$ (2001) Tumor necrosis factor- $\alpha$ levels in long-term marrow cultures from patients with aplastic anemia: modulation by granulocyte-macrophage colony-stimulating factor. Am J Hematol 68: 144-148.

23. Zoumbos NC, Gascon P, Djeu JY, Young NS (1985) Interferon is a mediator of hematopoietic suppression in aplastic anemia in vitro and possibly in vivo. Proc Natl Acad Sci USA 82: 188-192.

24. Tianshun L, Zhiping Z (1991) Interleukin-2 (IL-2) production and interleukin-2 receptor expression in patients with aplastic anemia. Chinese $\mathrm{J}$ Cancer Res 3: 70-73

25. Solomou EE, Keyvanfar K, Young NS (2006) T-bet, a Th1 transcription factor is up-regulated in T cells from patients with aplastic anemia. Blood 107: 39833991.

26. Gascon P, Zoumbos NC, Scala G, Djeu JY, Moore JG, et al. (1985) Lymphokine Abnormalities in Aplastic Anemia: Implications for the Mechanism of Action of Antithymocyte Globulin. Blood 65:407-413.

27. Raefsky EL, Platanias LC, Zoumbos NC, Young NS (1985) Studies of interferon as a regulator of hematopoietic cell proliferation. J Immunol 135: 2507-2512.

28. Selleri C, Sato T, Anderson S, Young NS, Maciejewski JP (1995) Interferongamma and tumor necrosis factor-alpha suppress early and late stages of hematopoiesis in vitro and induce programmed cell death. Blood 165: 538-546.

29. Maciejewski JP, Selleri C, Sato T, Anderson S, Young NS (1995) Increased expression of Fas antigen on bone marrow CD34+ cells of patients with aplastic anaemia. Br J Haematol 91: 245-252.

30. Nagafuji K, Shibuya T, Harada M, Mizuno S, Takenaka K, et al. (1995) Functional Expression of Fas Antigen (CD95) on Hematopoietic Progenitor Cells. Blood 86: 883-889.

31. Luther-Wyrsch A, Nissen C, Wodnar-Filipowicz A (2001) Intracellular Fas ligand is elevated in $\mathrm{T}$ lymphocytes in severe aplastic anaemia. $\mathrm{Br} \mathrm{J}$ Haemato 114: 884-890.

32. Callera F, Falc RP (1997) Increased apoptotic cells in bone marrow biopsies from patients with aplastic anaemia. $\mathrm{Br} \mathrm{J}$ Haematol 98:18-20.

33. Risitano AM, Maciejewski JP, Green S, Plasilova M, Zeng W, et al. (2004) Invivo dominant immune responses in aplastic anaemia: molecular tracking of putatively pathogenetic T-cell clones by TCR beta-CDR3 sequencing. Lancet 364: 355-364.

34. Katagiri T, Sato-Otsubo A, Kashiwase K, Morishima S, Sato Y, et al. (2011) Frequent loss of HLA alleles associated with copy number-neutral $6 \mathrm{pLOH}$ in acquired aplastic anemia. Blood 118: 6601-6609.

35. Afable li MG, Wlodarski M, Makishima H, Shaik M, Sekeres MA, et al. (2011) SNP array-based karyotyping: differences and similarities between aplastic anemia and hypocellular myelodysplastic syndromes. Blood 117: 6876-6884.

36. Zaimoku Y, Takamatsu H, Hosomichi K, Ozawa T, Nakagawa N, et al. (2017) Identification of an HLA class I allele closely involved in the autoantigen presentation in acquired aplastic anemia. Blood 129: 2908-2916.
37. Young NS, Bacigalupo A, Marsh JC (2010) Aplastic Anemia: Pathophysiology and Treatment. Biol Blood Marrow Transplant 16: S119-S125.

38. Gupta V, Eapen M, Brazauskas R, Carreras J, Aljurf M, et al. (2010) Impact of age on outcomes after bone marrow transplantation for acquired aplastic anemia using HLA-matched sibling donors. Haematologica 95: 2119-2125.

39. Townsley DM, Scheinberg P, Winkler T, Desmond R, Dumitriu B, et al. (2017) Eltrombopag Added to Standard Immunosuppression for Aplastic Anemia. N Engl J Med 376: 1540-1550.

40. Jilg W, Hannig K (1981) Lymphocyte surface proteins recognized by an antithymocyte-globulin. Hoppe Seylers Z Physiol Chem 362: 1475-1485.

41. Preville X, Flacher M, LeMauff B, Beauchard S, Davelu P, et al. (2001) Mechanisms involved in antithymocyte globulin immunosuppressive activity in a nonhuman primate model. Transplantation 71: 460-468.

42. Haidinger M, Geyeregger R, Poglitsch M, Weichhart $T$, Zeyda M, et al. (2007) Antithymocyte Globulin Impairs T-Cell/Antigen-Presenting Cell Interaction: Disruption of Immunological Synapse and Conjugate Formation. Transplantation 84:117-121.

43. Ayuk FA, Fang L, Fehse B, Zander AR, Kröger N (2015) Antithymocyte globulin induces complement-dependent cell lysis and caspase-dependent apoptosis in myeloma cells. Exp Hematol 33: 1531-1536.

44. Matsuda S, Koyasu S (2000) Mechanisms of action of cyclosporine Immunopharmacology 47: 119-125.

45. Lublin DM, Atkinson JP (1989) Decay-Accelerating Factor: Biochemistry Molecular Biology, and Function. Annu Rev Immunol 7: 35-58.

46. Huang Y, Qiao F, Abagyan R, Hazard S, Tomlinson S (2006) Defining the CD59-C9 binding interaction. J Biol Chem 281: 27398-27404.

47. Kinoshita T, Fujita M (2016) Biosynthesis of GPI-anchored proteins: special emphasis on GPI lipid remodeling. J Lipid Res 57: 6-24.

48. Hillmen P, Bessler M, Mason PJ, Watkins WM, Luzzatto L (1993) Specifi'c defect in $\mathrm{N}$-acetylglucosamine incorporation in the biosynthesis of the glycosylphosphatidylinositol anchor in cloned cell lines from patients with paroxysmal nocturnal hemoglobinuria. Proc Natl Acad Sci U S A 90: 5272 5276.

49. Boumil RM, Lee JT (2001) Forty years of decoding the silence in X-chromosome inactivation. Hum Mol Genet 10: 2225-2232.

50. Takeda J, Miyata T, Kawagoe K, lida Y, Endo Y, et al. (1993) Deficiency of the GPI anchor caused by a somatic mutation of the PIG-A gene in paroxysmal nocturnal hemoglobinuria. Cell 73: 703-711.

51. Nafa K, Mason P, Hillmen P, Luzzatto L, Bessler M (1995) Mutations in the PIG-A gene causing paroxysmal nocturnal hemoglobinuria are mainly of the frameshift type. Blood 86: 4650-4655.

52. Hu R, Mukhina GL, Piantadosi S, Barber JP, Jones RJ, et al. (2005) PIG-A mutations in normal hematopoiesis. Blood 105: 3848-3854.

53. Krawitz PM, Höchsmann B, Murakami Y, Teubner B, Krüger U, et al. (2013) A case of paroxysmal nocturnal hemoglobinuria caused by a germline mutation and a somatic mutation in PIGT. Blood 122: 1312-1315.

54. Luzzatto L (2013) PNH from mutations of another PIG gene. Blood 122: 1099 1100.

55. Araten DJ, Nafa K, Pakdeesuwan K, Luzzatto L (1999) Clonal populations of hematopoietic cells with paroxysmal nocturnal hemoglobinuria genotype and phenotype are present in normal individuals. Proc Natl Acad Sci U S A 96: 5209-5214.

56. Gargiulo L, Papaioannou M, Sica M, Talini G, Chaidos A, et al. (2013) Glycosylphosphatidylinositol-specific, CD1d-restricted $\mathrm{T}$ cells in paroxysmal nocturnal hemoglobinuria. Blood 121: 2753-2761.

57. Luzzatto L, Bessler M, Rotoli B (1997) Somatic Mutations in Paroxysmal Nocturnal Hemoglobinuria: A Blessing in Disguise? Cell 88: 1-4

58. Chen G, Kirby M, Zeng W, Young NS, Maciejewski JP (2002) Superior growth of glycophosphatidy linositol-anchored protein-deficient progenitor cells in vitro is due to the higher apoptotic rate of progenitors with normal phenotype in vivo. Exp Hematol 30: 774-782.

59. Araten DJ, Bessler M, McKenzie S, Castro-Malaspina $\mathrm{H}$, Childs $\mathrm{BH}$, et al. (2012) Dynamics of hematopoiesis in paroxysmal nocturnal hemoglobinuria 
Citation: Kaastrup K, Grønbæk K, Hadrup SR, Glenthøj A (2018) Autoimmune Processes as an Important Parameter for the Pathogenesis of Aplastic Anemia and Paroxysmal Nocturnal Hemoglobinuria. J Blood Lymph 8: 215. doi:10.4172/2165-7831.1000215

Page 8 of 8

(PNH): no evidence for intrinsic growth advantage of PNH clones. Leukemia 16: $2243-2248$

60. Hanaoka N, Kawaguchi T, Horikawa K, Nagakura S, Mitsuya H, et al. (2006) Immunoselection by natural killer cells of PIGA mutant cells missing stressinducible ULBP. Blood 107: 1184-1191.

61. Joyce S, Woods AS, Yewdell JW, Bennink JR, De Silva AD, et al. (1998) Natural Ligand of Mouse CD1d1: Cellular Glycosylphosphatidylinositol. Science 279: 1541-1544.

62. Broxmeyer HE, Christopherson K, Hangoc G, et al. (2012) CD1d expression on and regulation of murine hematopoietic stem and progenitor cells. Blood 119: $5731-5741$.

63. de Latour RP, Schrezenmeier H, Bacigalupo A, Blaise D, de Souza CA, et al. (2012) Allogeneic stem cell transplantation in paroxysmal nocturnal hemoglobinuria. Haematologica 97: 1666-1673.

64. Hillmen P, Young NS, Schubert J, Brodsky RA, Socié G, et al. (2006) The Complement Inhibitor Eculizumab in Paroxysmal Nocturnal Hemoglobinuria. $\mathrm{N}$ Engl J Med 355: 1233-1243.

65. Kaushansky K, Lichtman MA, Beutler E, Kipps TJ, Seligsohn U, et al. (2017) Williams Hematology (8th edn).

66. Dmytrijuk A, Robie-Suh K, Cohen MH, Rieves D, Weiss K, et al. (2008) FDA report: eculizumab (Soliris) for the treatment of patients with paroxysmal nocturnal hemoglobinuria. Oncologist 13: 993-1000.
67. Scheinberg P, Marte M, Nunez O, Young NS (2010) Paroxysmal nocturnal hemoglobinuria clones in severe aplastic anemia patients treated with horse anti-thymocyte globulin plus cyclosporine. Haematologica 95: 1075-1080.

68. Socié G, Mary JY, de Gramont A, Rio B, Leporrier M, et al. (1996) Paroxysmal nocturnal haemoglobinuria: long-term follow-up and prognostic factors. French Society of Haematology. Lancet 348: 573-577.

69. Afable MG, Tiu RV, Maciejewski JP (2011) Clonal evolution in aplastic anemia Hematol Am Soc Hematol Educ Progr 2011: 90-95.

70. Kulasekararaj AG, Jiang J, Smith AE, Mohamedali AM, Mian S, et al. (2014) Somatic mutations identify a subgroup of aplastic anemia patients who progress to myelodysplastic syndrome. Blood 124: 2698-2704.

71. Yoshizato T, Dumitriu B, Hosokawa K (2015) Somatic Mutations and Clonal Hematopoiesis in Aplastic Anemia. N Engl J Med 373: 35-47.

72. Hansen JW, Westman MK, Sjö LD, Saft L, Kristensen LS, et al. (2016) Mutations in idiopathic cytopenia of undetermined significance assist diagnostics and correlate to dysplastic changes. Am J Hematol 91: 1234-1238.

73. O'Keefe C, McDevitt MA, Maciejewski JP (2010) Copy neutral loss of heterozygosity: a novel chromosomal lesion in myeloid malignancies. Blood 115: 2731-2739.

74. Sloand EM, Rezvani K (2008) The Role of the Immune System in Myelodysplasia: Implications for Therapy. Semin Hematol 45: 39-48. 